Revue

Revue de l'histoire des religions

de Ihistoire

des religions

$3 \mid 2018$

Varia

Théâtre et chansons dans une Réforme en devenir (Suisse, 1530-1535). Des pratiques d'écriture au service d'une communauté à construire

Theatre and Songs in an Emerging Reformation (Switzerland, 1530-1535).

Practices of Writing in the Service of a Community under Construction

\title{
Geneviève Gross
}

\section{OpenEdition}

Journals

Édition électronique

URL : http://journals.openedition.org/rhr/8932

DOI : $10.4000 /$ rhr.8932

ISSN : 2105-2573

Éditeur

Armand Colin

Édition imprimée

Date de publication : 1 septembre 2018

Pagination : 415-450

ISBN : 978-2-200-93188-9

ISSN : 0035-1423

Référence électronique

Geneviève Gross, «Théâtre et chansons dans une Réforme en devenir (Suisse, 1530-1535). Des

pratiques d'écriture au service d'une communauté à construire », Revue de l'histoire des religions [En ligne], 3 | 2018, mis en ligne le 01 septembre 2020, consulté le 15 janvier 2021. URL : http:// journals.openedition.org/rhr/8932; DOI : https://doi.org/10.4000/rhr.8932 


\section{Théâtre et chansons dans une Réforme en devenir (Suisse, 1530-1535) \\ Des pratiques d'écriture au service d'une communauté à construire}

Théâtre et chansons font partie des moyens de diffusion des idées nouvelles. Ils accompagnent et soutiennent l'action pastorale qui mène à la conversion d'une ville. Porteurs de pratiques, ils débouchent certes sur un geste de performance s'ancrant dans l'espace communautaire, mais témoignent avant tout d'un acte d'écriture d'origine pastorale et sont dans leur forme des médias connus et usités de la chrétienté médiévale. Or, dans cette Réforme en devenir, le prédicant se trouve lui-même en voie de définition. Dès lors, théâtre et chansons, comme lieu d'écriture, rentreraient dans les enjeux de la Réforme, ceux de se défaire d'une culture religieuse et d'en mettre en place une nouvelle, construite sur une figure d'autorité, le prédicant, dispensateur de la cène et de la Parole de Dieu.

\section{Theatre and Songs in an Emerging Reformation (Switzerland, 1530-1535). Practices of Writing in the Service of a Community under Construction}

Theatre and songs were among the means by which new ideas were diffused. They accompanied and supported pastoral action, which would lead to the conversion of a city. As vehicles of practice, they gave rise, of course, to a performative act anchored in communal space, but above all they testified to an act of writing with a pastoral origin and, in their form, were media that were known and used by medieval Christianity. However, in the emerging Reformation, the preacher himself was in the process of being defined. As such, theatre and song, as a space of writing, contributed to the goals of the Reformation, namely to dismantle one religious culture and to put into place a new one constructed upon an authority figure, the preacher, who would dispense the Eucharist and the Word of God. 
Prendre le théâtre et le chant comme objet d'étude à l'heure de l'infiltration des idées nouvelles et de l'implantation d'Églises dans l'espace francophone permet d'interroger de façon conjointe deux médias de diffusion orale de la Réforme mais aussi de se confronter aux mécanismes d'une écriture polémique ancrée dans des genres hérités du christianisme médiéval.

Dans ces premières décennies du $\mathrm{XVI}^{\mathrm{e}}$ siècle, théâtre et chant sont deux genres à part entière qui appartiennent à la littérature de propagande et de controverse, tantôt ouvertement anticléricale, tantôt transportant des avertissements aux luthériens et démasquant leurs erreurs, ou mettant à jour les abus de l'Église romaine ${ }^{1}$. Ils deviennent également le lieu d'une expression et d'un élan spirituels marqués par les aspirations réformées. Terrain propice d'une dénonciation mais aussi d'une exposition de la foi pouvant se faire performative, ils sont susceptibles de s'ancrer au cœur d'une variété d'espaces, de l'espace clos d'une assemblée, d'une famille jusqu'aux églises, parvis, rues et places publiques, favorisant ainsi une diffusion mobile et une infiltration vivante et dynamique des idées nouvelles. Ils s'immiscent dans l'espace communautaire et viennent en fragiliser son apparente unité, provoquant désunion, rixe et scandale. À Genève, entre mars 1533 et août 1534 , le chant provoque des échauffourées et débouche sur une interdiction par les autorités de la ville d'entonner certaines chansons, dont celles du recueil hautement polémique publiées par Pierre de Vingle, les Chansons nouvelles, que nous analyserons ci-dessous². Le

1. Pour les premières critiques de Martin Luther sous forme chantée en langue française, on peut se reporter aux recueils suivants : La balade des leutheriens avec la chanson, [Lyon, Jacques Moderne, 1525] ; Le Te Deum pour l'eglise Lutherienne: en françoys et en latin. Avec la Ballade et la Chanson d'yceulx Lutheriens tout de nouveau imprimé, augmenté et corrigé. Le Te Deum de Luther, [s. 1., s. n.], 1528.

2. Christian Grosse, Les rituels de la Cène: le culte eucharistique réformé à Genève (XVI ${ }^{e}$ et XVII siècles), Genève, Droz, 2008, p. 69. Plus tôt, la diffusion de la Réforme dans les villes allemandes a également connu le recours au chant polémique élaboré sur des airs profanes ou religieux connus, provoquant troubles et procès, voir entre autres : Patrice Veit, « Entre violence, résistance et affirmation identitaire. À propos du cantique de Luther "Erhalt uns Herr bei deinem Wort" aux XVI et XVII ${ }^{\mathrm{e}}$ siècles ", Religion und Gewalt. Konflikte, Rituale, Deutungen (1500-1800), Göttingen, Vandenhoeck \& Ruprecht, 2006, p. 268-303. Sur les 
chant comme le théâtre viennent soutenir l'action communautaire menant à la conversion d'une ville, offrant aux fidèles un bagage argumentaire et un ensemble d'instruments de propagande malléables au service de leur instruction et de leur émancipation d'un système dévotionnel jugé abusif ${ }^{3}$.

$\mathrm{Au}$ cœur de l'espace qui nous concerne, le territoire suisseromand, l'Église réformée entre 1520 et 1535 reste avant tout, et même avec l'adoption de la Réforme en 1530 par Neuchâtel, une Église largement «invisible» au sens institutionnel du terme. Elle se comprend dans sa virtualité, puisqu'elle est davantage une Église en gestation aux prises quasi quotidiennement avec une Église institutionnelle à laquelle elle s'oppose. Le rejet de l'Église romaine s'effectue par le bais d'interventions concrètes impliquant la communauté dans son entier. Ces actions sont autant

usages du chant dans les différents types de Réforme, protestantes et réformées, dans l'espace européen : Patrice Veit, «Le Chant, la Réforme et la Bible», Le temps des Réformes et la Bible, dir. Guy Bedouelle et Bernard Roussel, Paris, Beauchesne, 1989, p. 659-681. Jonathan Beck mentionne également le cas plus tardif de Toulouse en 1548 et l'interdiction de certaines chansons comme les « Dix commandements », ou les «Articles de la foi », présentes d'ailleurs toutes deux dans le recueil imprimé en 1533 par Pierre de Vingle, intitulé S'ensuyvent belles et bonnes chansons ». Voir Jonathan Beck, Théâtre et propagande aux débuts de la Réforme. Six pièces polémiques du Recueil La Vallière, Genève, Slatkine, 1986, p. 48 ; et Le chansonnier huguenot $d u X_{X I}$ siècle, éd. Henri-Léonard Bordier, Paris, Libraire Tross, 1870, 2 vol., XXVII, p. 426.

3. L'emploi du théâtre, exploitant notamment des thèmes et une dramaturgie bibliques est attesté à Zurich et à Berne dès les années 1520 et tout au long du $\mathrm{XVI}^{\mathrm{e}}$ siècle, grâce notamment aux productions de Nicolas Manuel ou encore de Jacob Rueff : Glenn Ehrstine, Theater, Culture, and Community in Reformation Bern, 1523-1555, Leiden, Brill, 2002. Dans le baillage d'Orbe, territoire gouverné en alternance par Berne et Fribourg, restée catholique, le théâtre est présent. Réformés comme catholiques y recourent pour défendre leurs positions dans les années menant à l'adhésion à la Réforme par un vote majoritaire de la communauté, à l'image de l'année 1549: Guillaume de Pierrefleur, Mémoire de Pierrefleur, éd. Louis Junod, Lausanne, La Concorde, 1933, p. 177-178 ; Jonathan Beck, « La mise en scène de l'évangélisme militant (et clandestin) vers 1533-1535. Les moralités de La Maladie de Chrestienté et de La Verité cachée publiées par Pierre de Vingle à Neuchâtel », Les imprimés réformés de Pierre de Vingle (Neuchâtel, 1533-1535), dir. D. Desrosiers-Bonin et W. Kemp, Littératures, n 24, 2007, p. 181-220. Pour une étude sur l'usage du théâtre et plus particulièrement la tragédie biblique, les modalités de l'écriture théâtrale et son recours par la Réforme dans les années postérieures à notre chronologie : Ruth Stawarz-Luginbühl, Un théâtre de l'épreuve: tragédies huguenotes en marge des guerres de religion en France 1550-1573, Genève, Droz, 2012 ; Corinne Meyniel, De la Cène à la scène : La tragédie biblique en France pendant les guerres de religions (1550-1625), thèse sous la dir. de Christian Biet, Université de Paris Ouest Nanterre, 2010. 
d'occupations raisonnées de l'espace, de destructions ritualisées et de gestes porteurs d'enjeux symboliques et heuristiques ${ }^{4}$. Ainsi, l'installation de la Réforme à Genève est lue par Christian Grosse comme le résultat d'une série d'actes successifs, complémentaires et surtout collectifs, visant au «renversement de l'autel»c'està-dire à l'abolition du système rituel traditionnel. C'est pourquoi les premiers paroissiens convertis et convaincus, avec l'aide ou non des prédicants, investissent notamment le bâtiment même de l'église, venant dégrader son mobilier et ses cloches, renverser les tabernacles, piétiner les hosties et abattre les autels, lieu symbolique de la consécration eucharistique et de la puissance cléricale portée et revendiquée par un prêtre sacrificateur qu'ils rejettent. Recourant au langage de la spiritualité du Moyen Âge, ils entendent s'en détourner. Quittant ainsi une culture religieuse en la vidant de son sens pour avancer vers une nouvelle culture religieuse, ils participent à la substitution d'un rituel centré jusque-là sur la messe par une liturgie fondée et orientée sur la prédication et la cène ${ }^{5}$.

Or, on peut comprendre l'utilisation de genres spécifiques d'écriture comme un écho à ce procédé raisonné de renversement présent dans l'espace communautaire par l'investissement de lieux et la destruction d'objet précis. Le recours au théâtre et aux chansons entrerait en somme dans la finalité même de cette Réforme en devenir, celle de se défaire d'une culture religieuse héritée de la chrétienté médiévale. Ensemble, théâtre et chant représentent en effet un genre attaché à la culture orale et à la piété populaire. Pièces de théâtre et chansons se rattachent aux Mystères et autres pièces du théâtre religieux montées et jouées lors de festivités diverses, mais aussi aux réseaux des confréries et à leurs activités dévotionnelles. Les confréries ont d'ailleurs très

4. À ce propos, voir entre autres les travaux d'Olivier Christin et de Denis Crouzet autour de l'iconoclasme, de la violence religieuse et des guerres de religions en France : Olivier Christin, Une révolution symbolique : l'iconoclasme huguenot et la reconstruction catholique, Paris, Les Éd. de Minuit, 1991 ; Denis Crouzet, Les guerriers de Dieu: la violence au temps des troubles de religion (vers 1525-vers 1610), Seyssel, Champ Vallon, 1990 ; D. Crouzet, «Sur les symboliques du refus de la "farce" papiste », La Réforme en France et en Italie. Contacts, comparaisons et contrastes, études réunies par Philip Benedict, Silvana Seidel Menchi et Alain Tallon, Rome, École française de Rome, 2007, p. 403-430.

5. Ch. Grosse, Les rituels de la Cène, p. 43-114. 
largement contribué à l'essor de la lyrique religieuse en langue vulgaire grâce aux mouvements des laudesi ou des pénitents. Ces associations de dévotion laïque se chargent dès le XIII siècle de composer et de chanter des laudes, sorte de ballade religieuse avec refrain, le soir et les jours de fête dans une église déterminée. Elles célèbrent ainsi les joies et les douleurs de la Vierge, commémorent les saints patrons et font de la Passion du Christ le thème central de leur répertoire ${ }^{6}$. Théâtre et chant deviennent le symbole d'une écriture dévotionnelle attachée à l'intercession de la Vierge et des Saints mais surtout d'une activité pédagogique et vulgarisatrice adepte de l'entremêlement des genres et des récits légendaires, hagiographiques et scripturaires que développent les acteurs de la spiritualité médiévale ${ }^{7}$. Outils d'encadrement des fidèles, théâtre et chant sont plus largement des types d'écriture en lien avec le prêche ou du moins marqués d'une dimension pastorale signant une volonté d'édification morale de la communauté et venant interroger le laïc, acteur ou chanteur, dans sa part de transmission d'un contenu de foi et de participation à la pastorale ${ }^{8}$. Ils ont contribué à impliquer le fidèle sans dépasser toutefois l'interdiction de la prédication laïque. Dès lors, l'investissement du théâtre et du chant à l'heure de l'implantation des idées nouvelles dans l'espace francophone chercherait à réformer, à vider de leur sens et à nettoyer les formes mêmes de l'enseignement issues de la chrétienté médiévale et mènerait à une réappropriation des pratiques de vulgarisation, d'édification communautaire et d'exposition de la foi promues jusque-là par l'Église romaine.

6. André Vauchez, «La Bible dans les confréries et les mouvements de dévotion », Le Moyen Âge et la Bible, dir. Pierre Riché et Guy Lobrichon, Paris, Beauchesne, 1984 ; A. Vauchez, Les laïcs au Moyen Âge. Pratiques et experiences religieuses, Paris, Cerf, 1987 ; Catherine Vincent, Les confréries médiévales dans le Royaume de France, XIII-XVe siècle, Paris, Albin Michel, 1994.

7. Sur la tradition de l'écriture et de la représentation des Mystères, média oral ou « Bible animée », voyant l'entremêlement des genres mais aussi des textes entre récits bibliques et récits moins canoniques: Le temps des Réformes et la Bible, dir. Guy Bedouelle, Bernard Roussel, Paris, Beauchesne, 1989, p. 34-38 ; Graham A. Runnhalls, Études sur les Mystères. Un recueil de 22 études sur les mystères français, suivi d'un répertoire du théâtre religieux français du Moyen Age et d'une bibliographie, Paris, Champion, 1998.

8. Pour une réflexion autour des performances théâtrales, de l'art de la prédication et de la place du laïc au Moyen Âge dans la pastorale : Prédication et performance $d u X^{e}{ }^{e}$ au XVI $I^{e}$ siècle, 23 et 24 juin 2011, études réunies par Marie Bouhaïk-Gironès et Marie Anne Polo de Beaulieu, Paris, Classiques Garnier, 2014. 
L'espace francophone et plus particulièrement suisse-romand offre un cas d'étude à la perspective adoptée ici. En effet, sur le terrain de l'actuelle Suisse romande, la Réforme est en marche, puisque le territoire connaît dès la fin des années 1520 une infiltration et une circulation des idées « luthériennes » qui doivent beaucoup aux passages successifs à la Réforme de Zurich, Berne et Bâle ainsi qu'à un État savoyard affaibli dans son assise politique et son influence ${ }^{9}$. Cet espace allant des possessions partagées depuis 1476 entre Berne et Fribourg jusqu'aux rives du Léman, le Chablais et les villes épiscopales de Lausanne et Genève voit l'incursion répétée de prédicateurs d'origine française ${ }^{10}$. Au bénéfice de la protection de la ville de Berne, ville ouverte dès 1522 à une prédication de l'Évangile et passée en 1528 à la Réforme de type zwinglien, ces derniers, Guillaume Farel en tête, travaillent à la conquête d'un lieu public de la prédication, suscitant et animant des assemblées. Toutefois, les rôles liés à l'office pastoral restent encore à définir. Sur le terrain de l'évangélisation, les prédicants se confrontent aux prêtres, vicaires, curés, aux prêcheurs, dominicains ou franciscains, médiateurs consacrés et détenteurs expérimentés et reconnus d'une parole autorisée par leur Ordre, mais aussi aux instances épiscopales ou aux autorités d'une ville. Dans leurs démarches d'évangélisation, ils sont des sortes d'émissaires et de serviteurs de la ville de Berne, se faisant ambassadeurs des aspirations d'ordres spirituel et religieux nouveaux ${ }^{11}$. Par l'envoi de prédicants, Berne transcrit son engagement pour l'Évangile dans une rhétorique de service et

9. Sur l'implantation de la Réforme en Suisse et sa mise en place progressive, consulter notamment: Bruce Gordon, The Swiss Reformation, Manchester, Manchester University Press, 2002; Michael W. Bruening, Calvinism's First Battleground. Conflict and Reform in the Pays de Vaud, 1528-1559, Dordrecht, Springler, 2005.

10. À propos des premières tentatives de prédication à Lausanne et à Genève dans les années 1520 et 1530 ainsi que les succès des prédicateurs acquis à la Réforme à Aigle notamment ou encore Grandson et Neuchâtel, consulter : Charles Gilliard, "Les débuts de la réforme à Lausanne», Revue de théologie et de philosophie, t. 21, 1933, p. 249-273 ; Guillaume Farel : 1489-1565 : biographie nouvelle, Neuchâtel ; Paris, Delachaux \& Niestlé, 1930 ; Henri Vuilleumier, Histoire de l'Église réformée du Pays de Vaud sous le régime bernois, $4 \mathrm{t}$, Lausanne, La Concorde, 1927-1933.

11. Aimé-Louis Herminjard, Correspondance des Réformateurs dans les pays de langue française, t. 2, Genève, H. Georg ; Paris, Michel Levy, 1868, plus particulièrement les lettres 220, 262, 267, 269, 271. 
d'attention fraternelle à ses sujets, voisins, amis ou combourgeois, travaillant en somme avec les prédicants dans une perspective de nécessité chrétienne devant mener à la conversion de tous et donc au salut de tous. Lorsque le message porté est mal accueilli, en cas de plaintes, ou lors de procès menés contre eux, les prédicants en danger peuvent recourir à la ville de Berne. C'est dans ce cadre de loyautés et de soutiens que le prédicant existe. Il va alors chercher, dans l'investissement d'un territoire et d'une communauté dirigée et acquise institutionnellement parlant à l'Église traditionnelle, à défendre une légitimité de parole et d'action.

Dans ce contexte, la présence à Genève dès 1532 de l'imprimeur lyonnais Pierre de Vingle, beau-fils de Claude Nourry, et l'installation définitive de ses presses à Neuchâtel (1533-1535) constituent un tournant dans la diffusion de la Réforme en territoire francophone ${ }^{12}$. Les presses de Pierre de Vingle marquent en effet le passage à l'écrit de la contestation religieuse jusque-là largement orale. De l'emploi intensif de ce moyen novateur et moderne qu'est l'imprimerie résulte une vingtaine d'ouvrages. À cette entreprise éditoriale, sont associés Guillaume Farel engagé entre Aigle et Morat, le jeune Pierre Viret, Antoine Marcourt - actifs dans la région de Neuchâtel jusqu'à Genève -, Mathieu Malingre, prédicant à Yverdon, mais aussi l'hébraïsant cousin de Jean Calvin, Pierre Robert Olivétan, chargé de la traduction de la Bible $^{13}$. Engagés dans une dynamique de conversion sur un terrain d'activité particulier, ils prennent possession avec les presses de Pierre de Vingle d'un moyen de transmission supplémentaire de

12. À propos de la tentative d'installation des presses de Pierre de Vingle à Genève : Henri Delarue, "Olivétan et Pierre de Vingle à Genève : 1532-1533 », Bibliothèque d'Humanisme et Renaissance, t. 8, 1946, p. 105-118 ; Henri Naef, Les origines de la Réforme à Genève, t. 2, Genève, Librairie Alexandre Jullien, 1968 , p. 359.

13. Wiliam Kemp, «La redécouverte des éditions de Pierre de Vingle imprimées à Genève et à Neuchâtel (1533-1536) », Le livre évangélique en français avant Calvin, éd. Jean-François Gilmont et Wiliam Kemp, Turnhout, Brepols, 2004, p. 146-177 ; Les imprimés réformés de Pierre de Vingle, Neuchâtel, 1533-1536, éd. Diane Desrosiers-Bonin et William Kemp, Littératures, no 24 , 2 vol., 2007. Ce groupe de prédicants a reçu le qualificatif de groupe de Neuchâtel par les recherches récentes : D. Desrosier-Bonin, «L'Epistre de Marie d'Ennetières et les écrits du groupe de Neuchâel », "Ecrire et conter » : mélanges de rhétorique et d'histoire littéraire du XVI siècle offerts à Jean-Claude Moisan, éd. par MarieClaude Malenfant et Sabrina Vervacke, Laval, Presses de 1'Université Laval, 2003, p. 179-189. 
leurs revendications et deviennent par là-même auteurs. L'écriture devient alors un lieu à investir, non seulement pour diffuser des idées, mais également et surtout pour conquérir et asseoir une autorité pastorale devant mener à l'établissement d'une culture religieuse nouvelle, où le prédicant est appelé à devenir une pièce maîtresse par son rôle central d'enseignement et d'administrateur des sacrements au sein de la communauté. C'est dans ce sens que notre article interroge le geste d'écriture de ces prédicants et les modalités d'une mise en mots qui s'opèrent à travers l'emploi conscient de genres spécifiques : le théâtre et les chansons. Ainsi, dans la perspective d'analyse qui est la nôtre, nous analysons au sein du corpus vinglien les trois recueils de chansons ainsi qu'une des deux pièces de théâtre que comptent les productions de Pierre de Vingle, la Vérité cachée ${ }^{14}$.

\section{UNE VÉRITÉ USURPÉE : DU RENVERSEMENT D'UN SERVITEUR à la Création d'une communauté autour de la Parole}

Dans le courant de 1534, année de l'affaire des placards en France, et certainement autour du mois d'avril, sort des presses de Vingle à Neuchâtel une pièce de théâtre, la Vérité cachée $e^{15}$. Publiée sans nom d'auteur, cette pièce à six personnages se

14. La Verité cachée, devant cent ans faicte et composée à six personnages : nouvellement corrigée et augmentée avec les autoritez de la saincte escripture. Verité. Ministre. Peuple. Aucun. Avarice. Simonie, [Neuchâtel, Pierre de Vingle, 1533] ; S'ensuyvent plusieurs belles et bonnes chansons que les chrestiens peuvent chanter en grande affection de coeur; pour et affin de soulager leurs esperitz et de leur donner repos en Dieu [Neuchâtel, P. de Vingle, 1533] ; Chansons nouvelles demonstrantz plusieurs erreurs et faulsetez desquelles le povre monde est remply, par les ministres de Satan, [Neuchâtel, P. de Vingle, 1534]; Noelz nouveaulx. Musiciens amateurs des Cantiques, Au nom de Dieu, chantez noelz nouveaulx, Lesquelz sont faictz sur les vieulx et antiques: Je vous supply, delaissez les lubriques: Ne chantez point brayant comme noz veaulx, Glorieux chantres, ne vault point deux naveaux Recordez vous que Dieu veult l'humble cœur En foy contrict. Note cela chanteur, [Neuchâtel, P. de Vingle, 1534].

15. Nous savons que la pièce de la Vérité cachée a été représentée en 1563 dans le Nord de la France : Katell Lavéant, «Le théâtre du Nord et la Réforme : un procès d'acteurs près de Lille en 1563 », European Medieval Drama, 11, 2007, p. 59-77 ; Alain Lottin, « Un précieux témoignage dans la diffusion du calvinisme dans la châtellenie de Lille : La Farce de Mouvaux (1563)», Annales du comité flamand de France, 61, 2003, p. 155-161. Il est difficile de dire si la Vérité cachée a été portée sur scène auparavant. 
développe autour de deux convocations à assister au prêche et de deux prédications. Elle met en scène un Ministre chargé d'appeler une collectivité de fidèles, le Peuple, destiné à s'assembler autour de la chaire et à écouter deux prédications. La pièce se construit et se développe autour de l'acte de prédication publique et communautaire, un geste central et fondateur dans l'identité et l'existence des premières communautés réformées. Elle s'ouvre sur le prêche de Vérité et se ferme sur la prédication de Simonie vêtue de la robe de Vérité qu'elle a dérobée plus tôt. Ce vol ou cette usurpation de la tunique de Vérité résume l'essentiel du drame de la pièce, celle d'une vérité cachée et finalement retrouvée. Au renversement de Vérité, par le vol de sa tunique organisé par les serviteurs d'un Ministre qui se prend pour un seigneur, s'ajoute l'asservissement du Peuple qui se met au service non de la vérité mais d'un faux-maître, qui devrait, quant à lui, être à son service en l'édifiant et en lui enseignant la vérité.

La taxinomie choisie pour les personnages de la pièce participe du drame qui va se jouer, puisque Ministre désigne bel et bien un serviteur dans le langage du $\mathrm{XvI}^{\mathrm{e}}$ siècle. Très vite se distingue sous les traits de ce serviteur, un prêtre et clerc, porteur et demandeur d'autorité, désireux d'un Peuple qui doit, dit-il, «me croire» plutôt que de s'arrêter à Vérité $e^{16}$. Ministre, réfractaire à la vie de charité et de pauvreté que lui dessine et promet Vérité dans sa prédication, s'étonne d'ailleurs qu'elle le prenne pour un simple serviteur. Il se définit, quant à lui, en maître et chef du Peuple et refuse de se gouverner en mendiant. Il finit par s'en remettre à Avarice et à sa sœur Simonie devant les perspectives de richesses et d'honneur qu'elles lui prédisent. Il fait alors de Simonie la maîtresse de sa maison ${ }^{17}$. Avarice, l'appât du gain, quant à elle, y détiendra les clés, présentant dès lors par un jeu de mots

16. La Verité cachée, devant cent ans faicte et composée à six personnages : nouvellement corrigée et augmentée avec les autoritez de la saincte escripture. Verité. Ministre. Peuple. Aucun. Avarice. Simonie, [Neuchâtel, P. de Vingle, 1533], f. $\mathrm{C} 5 \mathrm{r}^{\circ}$. Le texte de la pièce sera prochainement publié et présenté dans une édition critique par Jonathan Beck: La maladie de Chrestienté et La verité cachée. Deux moralités de polémique religieuse imprimées par Pierre de Vingle (Neuchâtel, 1533-34), éd. Jonathan Beck, Estelle Doudet and Anlan Hindley, Paris, Garnier, à paraître. Dans l'attente de cette publication, nous faisons référence à la foliotation d'origine.

17. Ibid., f. C6r ${ }^{\circ}$. 
l'acquisition tarifée du salut par les œuvres ${ }^{18}$. Ces intronisations d'Avarice et de Simonie en maîtresse et gardienne des clés de la maison du Ministre conduiront par la suite à la disparition de Vérité et au vol de sa robe. Rentre alors dans la trame dramaturgique de la pièce l'imagerie de la maison, conduite non seulement par un maître, mais servie également par un certain nombre de serviteurs. Elle interroge non seulement l'autorité, sa nature et son exercice, mais explore également la notion de service. La pièce de la Vérité cachée investit dès lors une finalité : démasquer le serviteur devenu maître et la vérité déguisée. À ce dévoilement participe Aucun, ce singulier isolé du Peuple pour servir la trame narrative et dramatique de la pièce. Amené par Peuple au prêche de Vérité et participant à sa joie d'aller l'entendre, il s'en distinguera de plus en plus. Ce quelqu'un, traduit en langage actuel, qu'on verra travailler aux côtés du Peuple, apparaît comme un homme sans distinction ou titre particulier. Il illustre en somme un accueil favorable de la Vérité, qui avait prédit lors de son prêche : "Qui est de Dieu, ma voix escoute; Qui n'est de Dieu jamais n'en gouste $»^{19}$. Aucun se joue de l'autorité de Ministre, défendue et construite sur ses titres de docteur en théologie, car il dit désormais ne craindre « jamais reproche de tout le monde, que n'ensuyve la Verité, tant que je vive $»^{20}$. Il fait de Vérité sa maîtresse et détient par elle liberté d'esprit.

Dès lors, tout l'enjeu et l'intérêt de la pièce se trouvent dans les personnages de Ministre et d'Aucun, dans l'expression et la nature des liens respectifs qu'ils entretiennent et développent avec Peuple. Malgré les efforts d'Aucun, le Peuple va affermir son lien au Ministre, lui donnant du «monseigneur », du « sire» et du «prudent seigneur $»^{21}$. Il le reconnaît comme «son prélat», son supérieur, ne voulant point de débat sur ce point et promet de « vivre comme mes anciens » n'ayant que faire de la nouveauté22. Est ainsi mis en scène l'endurcissement des cœurs que dénonce dès 1530 l'ouvrage dû à Farel et Viret, De la Tressaincte Cene. À vouloir maintenir ce que les hommes ont mêlé et changé aux
18. Ibid., f. C5r ${ }^{\circ}$.
19. Ibid., f. A2 $\mathrm{r}^{\circ}$.
20. Ibid., f. $\mathrm{B} 7 \mathrm{r}^{\circ}-\mathrm{v}^{\circ}$.

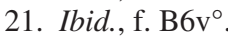
22. Ibid., f. B8r ${ }^{\circ}$. 
ordonnances de Dieu, les fidèles endurcissent leur cœur contre le Seigneur $^{23}$. Ils respectent, par crainte de la puissance de Ministre, des traditions humaines et cultivent un état de servilité.

Au sortir du prêche mi-latin mi-français de Simonie farci de ses recours elliptiques et orientés à la Sainte Écriture, le Peuple ânonne les paroles plusieurs fois répétées en chaire par cette Vérité déguisée. Il a retenu de la prédication cette parole : «forte alithes $»^{24}$. Avec cette idée au demeurant tout à fait incompréhensible, Aucun profite de faire des jeux de mots en grec et en hébreu ${ }^{25}$. Mais, il reprend bien plus le Peuple, lui redonnant les mots exacts de Simonie: «ferte arietes $»^{26}$. Il poursuit disant que cette prétendue Vérité ment dès ses premiers mots. D'une part, cette Vérité situe mal le passage sur lequel elle appuie son prêche. D'autre part, le texte hébreu ne parle pas de « petits béliers », mais dit bien autre chose. Aucun cite alors le passage dans son entier: «Ô princes! Au dieu éternel. Donnez, donnez gloire et honneur au Dieu éternel créateur $»^{27}$. Cette fausse Vérité a ainsi détourné l'Écriture pour servir la gloire des hommes. Aucun est là pour avertir le Peuple et déjouer cette autorité prise sur l'Écriture. Mieux, Aucun, par sa connaissance du grec et de l'hébreu, tente de sortir le Peuple de son état d'ignorance. Ces emplois du grec et de l'hébreu dans la bouche d'Aucun ne sont pas sans rappeler l'utilisation de ces langues par la «pauvre créature » que met en lumière le Petit Traicté d'Antoine Marcourt ${ }^{28}$. Dans cet ouvrage sorti des presses de Pierre de Vingle en 1534, cette "pauvre créature » affronte ouvertement l'interdiction des théologiens de la Sorbonne d'employer l'hébreu et le $\operatorname{grec}^{29}$. Elle y recourt par deux fois ${ }^{30}$. Par ce biais, au contraire de ces docteurs,

23. [Guillaume Farel], De la Tressaincte Cene de nostre Seigneur Jesus : et de la Messe qu'on chante communement, publié et introduit par Francis M. Higman : «Les débuts de la polémique contre la messe : De la tressaincte cene de nostre seigneur et de la messe qu'on chante communement " ( $1^{\mathrm{re}}$ éd., 1987), Lire et découvrir : la circulation des idées au temps de la Réforme, Genève, Droz, 1998, p. $251-252$ (f. $\mathrm{A} 2 \mathrm{r}^{\circ}-\mathrm{A} 3 \mathrm{v}^{\circ}$ ).

24. Ibid., f. E3r ${ }^{\circ}$.

25. Ibid., f. $\mathrm{E} 3 \mathrm{v}^{\circ}$.

26. Ibid.

27. Ibid.

28. [Antoine Marcourt], Petit traicté tres utile, et salutaire de la saincte eucharistie de nostre Seigneur Jesuchrist, [Neuchâtel, P. de Vingle], 1534.

29. Ibid., f. AIIIr ${ }^{\circ}$.

30. Ibid., f. $\mathrm{AIVr}^{\circ}$. 
elle entend se montrer amie des bonnes lettres, autrement dit des Écritures, de leur connaissance et de leur exposition dans une pureté retrouvée. Dès lors, avec ce bagage, elle ne craint plus de s'élever contre l'autorité des théologiens. Bien plus, elle s'autorise, en «pauvre créature » qu'elle est, à remplacer ces derniers et à enseigner à la communauté. Par leur interdiction même, les théologiens ont manifesté un renoncement flagrant et évident à remplir leur charge d'édification. La connaissance des langues et leur usage à des fins de dévoilement de traductions fautives et d'interprétations abusives permettent ici à Aucun d'endosser une autorité et de marquer une frontière grâce à laquelle la juridiction spirituelle cléricale est rejetée.

Aucun se met désormais au service de Vérité. Recourant à l'image de la maison et pour illustrer sans doute la charge que se donne Aucun, Vérité occupera une maison quelconque au cœur de la communauté ${ }^{31}$. C'est Aucun qui l'accueille chez lui et signe ainsi son intention de la protéger et surtout d'en faire la maîtresse de sa maison. Cet accueil au cœur de la communauté symbolise en somme un accès et par conséquent une transmission de la vérité détachée de l'institution romaine. Il se fait finalement l'écho du dialogue qui ouvre, dans la version imprimée du moins, la Vérité cachée et ancre le titre même de la pièce, La Vérité cachée, devant cent ans à cette chronologie d'un siècle passé semble-t-il sans vérité, pouvant inscrire la pièce dans le contexte lié au concile de Bâle et de Florence (1431-1439), et de la condamnation des positions de Jan Hus et des frères de Bohême. Ce prologue rédigé sous la forme d'un intermède dialogué est programmatique de l'intrigue de la pièce et de sa résolution. Un certain compagnon nommé « $\mathrm{C}$ » portant sous le bras un «vieil traicté », pourtant neuf d'apparence, éveille l'intérêt de «P». C'est bien en effet la caractéristique de ce traité, vraisemblablement élaboré cent ans plus tôt en Hainaut. Par ce biais, « $\mathrm{C}$ » invite l'intéressé « $\mathrm{P}$ » à aller sous la tente de Vérité et à connaître ce qu'il se devait de connaître. Sous cette tente, « $\mathrm{P} »$ se dit heureux, «car, dit-il, tout bien est ouvert Par Verité » mais aussi comme le précise « $\mathrm{C} »$ la

31. Dès la fin du sermon de Vérité, Aucun parle de la Vérité comme de sa maîtresse. Plus loin, il en fera la maîtresse de sa maison : La Verité cachée, f. $\mathrm{B} 7 \mathrm{v}^{\circ}, \mathrm{E}^{\circ} \mathrm{r}^{\circ}$. 
divulgation de tout mal est assuré. Grâce à l'action de « $\mathrm{C}$ » et à son invitation, cette vérité cachée depuis longtemps est désormais dévoilée, car cette vérité «latente, Nouvellement par moy tu la revois ». Par cette sorte de mise en abîme, est ainsi donné corps à l'attention fraternelle d'Aucun envers son prochain et à son rôle à venir, appelé à être témoin et gardien tant de l'histoire et des causes de l'abandon de Vérité que de sa restauration et de son habitation, sa «tente ».

L'identité d'Aucun se dessine également par l'évocation de saint Paul. Simonie, Avarice et Ministre craignent les résistances et les propos de ce «Saint Paul», mais les menaces d'une accusation et d'une excommunication restent vaines et sans effet ${ }^{32}$. Leur crainte va jusqu'à faire dire à Ministre: "Que ne se rompit il [Paul] le cou, Allors qu'il tomba du cheval, Il nous fait beaucoup plus de mal Que ne fait le nouveau turc $»^{33}$. C'est l'occasion pour Aucun de s'illustrer en connaisseur expert et fidèle aux sources scripturaires. Mentionnant explicitement les deux passages des Évangiles, aux Actes et chez saint Luc, de la conversion de Paul, Aucun se montre fin lecteur des Écritures et plaide pour l'absence de cheval. Par ce biais, il prend autorité sur un Ministre plus attaché au récit légendaire de la conversion de Paul, et à sa représentation courante depuis le XII ${ }^{\mathrm{e}}$ siècle, qu'aux Écritures ${ }^{34}$. L'identité d'Aucun se construit d'autant plus autour de Paul que Ministre dit vouloir renoncer à l'apôtre. Cet éloignement et la négation du Ministre servent à modeler une nouvelle communauté représentée par cet Aucun et à résoudre l'aporie de la continuité et de l'ancienneté fondatrice de l'autorité du clergé auprès de Peuple. De persécuteur des chrétiens, Paul est devenu disciple du Christ et a prêché l'abolition de la Loi, sans faire partie des douze apôtres et donc sans avoir connu le Christ avant sa mise à mort. Par une intense activité missionnaire et sous la menace violente

32. Ibid., f. $\mathrm{C} 2 \mathrm{v}^{\circ}$.

33. Ibid.

34. À partir des $\mathrm{XIII}^{\mathrm{e}}$-XIV ${ }^{\mathrm{e}}$ siècles, Paul est représenté sur le chemin de Damas en compagnie d'un cheval. Il est soit déjà tombé à terre, soit en train d'être désarçonné : François Boespflug, «La conversion de Paul dans l'art médiéval », Paul de Tarse. Congrès de l'ACFEB (Strasbourg, 1995), éd. Jacques Schlosser, Paris, Cerf, 1996, p. 147-168; Nicole Bériou, L'avénement des maîtres de la Parole : la prédication à Paris, t. 1, Paris, Institut d'études augustiniennes, 1998, p. 417. 
d'être persécuté, il a annoncé l'avènement du Christ sur terre pour le salut des hommes. Ainsi, cette communauté s'affirmerait dans une similitude de combat, d'action et de témoignage avec l'apôtre Paul $^{35}$. Elle cultiverait finalement une lecture de la réalité, celle de la confrontation, à l'heure d'une vérité à nouveau révélée, «aux successeurs des pharisiens », que dénonce notamment Farel dans le Manière et fasson ${ }^{36}$. Ces successeurs des pharisiens inquiètent par leur puissance les justes, «ne désirant que leur mort » et forcent par l'épée les fidèles à croire.

Autour du texte, on recense plus de 462 marginalia. Ces recours à l'autorité scripturaire ornent pour l'essentiel les propos de Vérité et d'Aucun. Ils viennent matériellement faire barrage et renverser la pratique de l'autorité défendue par Ministre et attribuée à l'Église romaine. Cette autorité est comprise comme une forme d'asservissement des fidèles devant se soumettre « aveuglément» non pas à la vérité évangélique mais avant tout à leur autorité, défendue notamment par l'ancienneté. Cette autorité est en péril d'autant que désormais, à suivre la trame dramaturgique de la pièce, Ministre fait face dans sa communauté à une maison du commun, lieu d'habitation quotidienne d'Aucun, devenue siège de Vérité par le geste protecteur de ce personnage sans distinction incarnant l'élection par les moindres. Les références bibliques sont citées par bloc accompagnant une ou plusieurs tirades, en ne s'attachant pas, le plus souvent, à un passage littéral et

35. C'est d'ailleurs avec ce même recours à l'apôtre Paul que Guillaume Farel, lors de la Dispute de Lausanne de 1536, conseille aux prêtres de délaisser leur ministère et la messe pour s'engager dans une activité pastorale tournée vers l'édification des fidèles : «Et conseil à tous prestres, qu'ilz retournent et qu'ilz regardent diligemment l'escripture. Et ceux auxquelz Dieu aura donné la grace de pouvoir enseigner, qu'ilz facent comme sainct Pol qui premièrement, destruisoit l'eglise, et, quand il fut converty, de tout son pouvoir l'edifioit, ainsi eux, comme ilz ont esté seduictz et ont seduict le peuple, se retournent à verité et edifient le peuple en la saincte foy et doctrine de Jesus ». Les actes de la Dispute de Lausanne : 1536, publiés intégralement d'après le manuscrit de Berne par Arthur Piaget, Neuchâtel, Secrétariat de l'Université, 1928, p. 200.

36. [Guillaume Farel], La maniere et fasson qu'on tient en baillant le sainct baptesme en la saincte congregation de Dieu : et en espousant ceulx qui viennent au sainct mariage, et à la saincte Cene de nostre seigneur, es lieux lesquelz Dieu de sa grace a visité, faisant que selon sa saincte parolle ce qu'il a deffendu en son eglise soit rejecté, et ce qu'il a commandé soit tenu. Aussi la maniere comment la predication commence, moyenne et finit, avec les prieres et exhortations qu'on faict à tous et pour tous, et de la visitation des malades, Neuchâtel, P. de Vingle, 1533 , f. AIIIr ${ }^{\circ}$. 
précis du discours. Dans leur majorité, elles servent de matériel à la formulation condensée d'une critique ou d'une position. La compilation fouillée des marginalia transcrit une exigence d'étude, passant par la recherche, la lecture et l'établissement d'un jugement quant à l'adéquation ou non des propos allégués à la vérité scripturaire. C'est bien à cet examen que le lecteur et le spectateur sont appelés et ce, par la bouche de Vérité qui leur demande de $«$ hanter les Ecritures $»^{37}$.

Une autorité autre est alors défendue et relayée par l'absence de nom d'auteur dans la publication de la Vérité cachée. Cette autorité, promue non par la dignité et le statut, est bien davantage acquise par l'exercice d'un jugement qu'illustre le jeu d'Aucun : la mise en examen et la dénonciation d'abus attestés et justifiables, donnant finalement à l'Écriture la place la plus haute, comme autorité seule au-dessus des hommes et de leurs honneurs. C'est pourquoi, si l'anonymat sert et concourt, il est vrai, à l'effacement de l'auteur pour des raisons sécuritaires liées à la censure, il pourrait bien plus détenir une dimension illustrative et entrer en résonance avec l'argumentaire présent dans la pièce. Par son écrit appelé à être joué et mis en scène, l'auteur resté anonyme défend une dynamique d'affirmation d'une autorité qui s'opère à l'écrit, comme à l'oral, dans la soumission, une soumission renversée puisqu'elle s'exerce dorénavant du fidèle et du lecteur envers le contenu d'une prise de parole et de l'auteur qui le porte. Par ce processus, la légitimité à édifier s'acquiert et se gagne. Dans cette perspective, la Vérité cachée viendrait, il est vrai, par le genre qu'elle représente, s'inscrire dans le prolongement du prêche par la résonance et le martèlement de certaines thématiques. Toutefois, elle est davantage une reproduction du prêche non pas du point de vue de son seul contenu, que de celui des circonstances et des conditions qu'entendent mettre en place les prédications réformées $^{38}$. En effet, dans la réédition en 1534 du Sommaire et

37. Ibid., f. A5 ${ }^{\circ}$.

38. Katell Lavéant s'est intéressée à la problématique du théâtre non seulement comme support et prolongement à la prédication mais aussi à la nature même de la Vérité cachée comme prédication : "Une pièce peut-elle être un prêche ? Le sermon dans une pièce protestante et sa représentation, 1533-1563 », Prédication et performance du XII au XVI siècle, Actes du colloque, Paris, 23 et 24 juin 2011, études réunies par Marie Bouhaïk-Gironès et Marie Anne Polo de Beaulieu, Paris, Classiques Garnier, 2014, p. 251-272. Sur le lien entre le prêche et les deux supports 
Brieve Déclaration que Farel publia une première fois en 1529 , une exhortation s'adresse à l'auditeur des prêches. Elle l'appelle en effet à vérifier les paroles du prédicant à la lumière de la Bible et à se faire entendre en cas de prédication de fausses doctrines ${ }^{39}$. À suivre cette exhortation, le fidèle devenu un familier et un connaisseur des Écritures se fait gardien de la vérité pour luimême et pour son salut mais aussi pour sa communauté. Autour de l'usage de textes sacrés et religieux, deux communautés, appelées à devenir le corps constitutif d'une nouvelle Église, et que nous pourrions appeler des communautés d'interprétation, se conçoivent dans leur capacité respective à recourir à la Parole de Dieu et dans l'autorité qui en découle. Leur recours est différencié, aux fidèles est laissée une responsabilité, celle de recevoir une édification conforme aux Écritures ; aux prédicants appartiennent l'édification et l'instruction des fidèles. Ces deux communautés, prédicants et fidèles, se constituent en temps réel dans l'instant de la prise de parole, orale ou écrite, et se reconnaissent mutuellement une autorité par et dans l'autorité des Écritures. À partir de ce point de ralliement s'envisage et se construit une communauté composée de fidèles rassemblés autour d'un prédicant, cet homme du commun dont ils examinent en même temps qu'ils valident la justesse de son enseignement.

Bien plus, la Verité cachée offre des outils d'identification et renforce cette dynamique par la dimension visuelle et surtout par les effets de la personnification propre à l'écriture polémique et théâtrale ${ }^{40}$. Par la réduction de la cléricature et de la prêtrise à des pratiques facilement identifiables, elle oriente le regard et souligne la volonté de circonscrire l'erreur. Elle accompagne et soutient le processus d'émancipation des fidèles décrits comme aveugles et aveuglés par les «enchanteries» des prêtres et la prise en

« pastoraux » du théâtre et du chant, se rapporter notamment à : Alan Hindley, «Preaching and plays : the sermon and the Late Medieval French Moralités », Le Moyen Français, revue d'études linguistiques et littéraires, 42, 1998, p. 71-85.

39. [Guillaume Farel], Summaire, et briefve declaration d'aucuns lieux fort necessaires a ung chascun Chrestien, pour mettre sa confiance en Dieu, et ayder son prochain. Item, ung traicté du Purgatoire nouvellement adjousté sur la fin, [Neuchâtel, P. de Vingle], 1534, f. HIVv $-\mathrm{HVV}^{\circ}$.

40. Mathilde Bernard, «La prosopopée dans la chanson polémique des guerres de religion », à paraître dans les actes du colloque Polémique en chanson $I V^{e}-X V I^{e}$ siècle, organisé par Luce Albert et Mickaël Ribreau, 11-13 juin 2015. 
main de leur destin spirituel. Plus précisément encore, elle vient soutenir la reconnaissance de la nature des prêcheurs. Le lecteur ou le spectateur, qui sera bientôt un auditeur de prédication, est désormais posé devant une réalité, celle de la nature contraire des prédications, tantôt vraie, car découlant de Vérité et tantôt voilée, abrutissante et mensongère, car formulée sous couvert de vérité à l'image de celle prononcée par Simonie, personnification du trafic des biens spirituels et maîtresse de la maison de Ministre. Posés devant un choix, celui d'une adhésion ou non à la vérité, le spectateur, le lecteur et le futur auditeur de prêches détiennent avec cette pièce un outil de discernement. Ce processus d'identification s'opère avant même le sermon fictif d'une Simonie déguisée en Vérité qui, dans une visée polémique d'ordre institutionnel - le rejet du prêtre - brode sur une thématique anticléricale fréquente, celle d'un latin clérical maladroit et altéré dans son sens, conduisant finalement à la lecture de la dignité sacerdotale comme exercice d'un pouvoir pris sur les Écritures et d'un pouvoir autoritaire et arbitraire sur les fidèles. À une identification rapide par le simple recours au latin, viennent s'ajouter la forme et la structure issues d'une pratique pastorale construite tout au long du Moyen Âge et adoptée pour la prédication par les figures pastorales courantes : frères mendiants et curés.

De ces artifices rhétoriques, l'auteur - au même titre que le prédicant qu'il est certainement - et sa prédication tirent finalement leur autorité. Auteur d'une prédication sans nul doute éloignée d'une structure élaborée sur le schéma des artes praedicantes, il s'assure par ce biais une légitimité. En prêche, il s'illustrera comme ce «prebstre qui raconte Tout l'evangile » sans en «faire mesconte », ayant «Dieu pour souverain», une figure que les derniers mots de la Vérité cachée exhortent d'ailleurs à suivre ${ }^{41}$. Proposant ainsi une prédication "prompte de Vérité », il sera en tout contraire au prêtre qui consacre «pour Argent, Dieu en pain ».

41. Dans ces mots finaux, le compagnon exhorte les lecteurs et les spectateurs à s'appliquer à « Éprouvez les esprits » pour savoir s'ils viennent de Dieu ou non et donne en outre une marche à suivre pour discerner ce qui est prêché, le faux ou la vérité : «Quand vous verrez ung prebstre qui raconte Tout l'evangile, et n'en faire mesconte, Et qu'il n'aura que Dieu pour souverain, Sans consacrer, pour l'argent, Dieu en pain, Alhors aurez predication prompte de Verité » : La Verité cachée, $\mathrm{E} 7 \mathrm{v}^{\circ}$. 
À partir de la distinction mise en scène et soutenue par le discours, s'enclenche un processus de détachement du fidèle face aux usages dévotionnels qu'il a connus et pratiqués jusque-là, processus qui mène in fine à une affiliation au prédicant et à son prêche reconnus désormais dans leur légitimé.

Dans la Vérité cachée, les interactions d'Aucun et du Ministre avec le Peuple dessinent, en interrogeant la notion de service à la Parole, à la communauté et à la vérité, les contours fondamentaux du rôle et de la place d'un nouveau médiateur du sacré au sein de la communauté. S' ancrant certainement dans le cadre d'une culture et d'une pratique d'écriture construite sur un patrimoine médiéval - le Mystère, la Moralité historique, la farce -, la Vérité cachée annonce une rupture, visible dans sa seule trame dramaturgique. Comme l'a noté Jean-Pierre Bordier dans son analyse de la pièce, on constate l'absence de personnages allégorisés tels que Justice et Miséricorde, venant toucher le spectateur dans ses attentes et ses habitudes ${ }^{42}$. Bien plus, le théâtre, lieu jusque-là d'une mise à nu du trompeur ou d'une lecture moralisante des épisodes de vies exemplaires des saints ou du Christ, servirait à l'affirmation d'un rapport rénové à la vérité et à son transmetteur. Il deviendrait, à l'échelle de la communauté, le lieu d'un dévoilement ou d'une révélation à des fins de renversement institutionnel. Ce nouveau rapport à la vérité surgit au cœur d'une communauté par le théâtre représenté. Par ce biais, en tableaux successifs, les figures d'un changement se matérialisent, autorisant à penser le renversement, à le dire et à le suivre. À cette dynamique de construction d'un lien neuf à la Vérité, participerait également, selon nous, la seconde pièce de la production vinglienne, la Moralité de la maladie de chrestienté, appelant ouvertement dans son titre à un genre théâtral connu devant mener à travers la mise en scène de personnages allégoriques à une leçon édifiante ${ }^{43}$. Dans sa trame dramaturgique et ses personnages symboliques, la pièce fait alors intervenir au

42. Jean-Pierre Bordier, « Découvrir La Vérité cachée », Théâtre et polémique religieuse, Regards croisés sur la scène européenne, http://umr6576.cesr.univtours.fr/publications/polemique/fichiers/pdf/02-BORDIER.pdf, Centre d'Études Supérieures de la Renaissance, 2014, p. 13.

43. Sur la moralité, sa définition et ses dérivés: Jonathan Beck, Théâtre et propagande aux débuts de la Réforme. Six pièces polémiques du Recueil La Vallière, Genève, Slatkine, 1986. 
chevet de cette chrétienté malade les trois vertus théologales: foi, espérance et charitét ${ }^{44}$. Dans quelle mesure, ces attributs traditionnellement attachés à une pratique de la lecture biblique héritée de la scolastique médiévale sont-ils réinvestis et sortis de leur usage théologique ? Dans quelle mesure, contribuent-ils à la défense d'un accès large à la vérité et participent-ils à l'élaboration d'un agir chrétien commun à tous, lié à une vie en chrétienté dans la foi et la charité, conduite par le Christ et menée dans l'espérance de son avènement et le respect des ordonnances d'un Évangile retrouvé dans sa pureté ?

\section{Trois recueils de chansons de Pierre de Vingle : DIRE ET TRANSMETTRE UNE FOI}

Entre 1533-1534, Pierre de Vingle imprime 3 recueils de chansons ${ }^{45}$. Cet ensemble de 48 chansons est constitué d'un cahier de 5 chansons polémiques formant un recueil à part entière ${ }^{46}$, puis d'un recueil de 19 chansons marqué d'un élan plus spirituel tourné

44. [Matthieu Malingre], Moralité de la maladie de Chrestienté, a .xiij. personnages : en laquelle sont monstrez plusieurs abuz, advenuz au monde, par la poison de peché et l'hypocrisie des hereticques. Foy. Esperance. Charité. Chrestienté. Bon auvre. Hypocrisie. Peché. Le medecin celeste. Inspiration. L'aveugle. Son varlet. L'apoticaire. Le docteur. Ceste moralité reprend Les abuz de Chrestienté, Celuy qui est en Christ enté Jamais à la mort ne mesprend, [Neuchâtel, Pierre de Vingle], 1533. Pour une étude récente sur ce texte : CharlesAntoine Chamay, "La moralité de maladie de chrestienté à XIII personnages" de Mathieu Malingre et la polémique religieuse », Le théâtre polémique français, 1450-1550, Rennes, Presses universitaires de Rennes, 2008, p. 179-187.

45. Ces trois recueils forment ce que Marc Honegger appelle dans sa thèse « le noyau des chansonniers huguenots »: M. Honegger, Les Chansons spirituelles de Didier Lupi et les débuts de la musique protestante en France au XVI siècle, Paris, 1970, p. 6. Ils ont été étudiés dans leurs dimensions d'outil de propagande par Ann Ullberg, «Les premières chansons imprimées par Pierre de Vingle : la chanson populaire au service de l'Évangile », Cinq siècles d'histoire religieuse neuchâteloise: approches d'une tradition protestante: actes du colloque de Neuchâtel (22-24 avril 2004), Neuchâtel, Université de Neuchâtel, 2009, p. 41-56 ; Geneviève Gross, "Songs and singing in developing Reformation. From a Scattered Community of Believers to a Visible Church (1530-1536) », "Church" at the time of the Reformation - Invisible community, visible parish, confession, building... ?, Göttingen, Vandenhoeck \& Ruprecht, à paraître.

46. Chansons nouvelles demonstrantz plusieurs erreurs et faulsetez desquelles le povre monde est remply, par les ministres de Satan, [Neuchâtel, P. de Vingle, 1534]. 
vers une foi christocentrée ${ }^{47}$, et enfin d'un dernier recueil de 24 chants exclusivement composés autour de la thématique de Noël ${ }^{48}$. Des paraphrases assez libres du psautier parsèment également les recueils. Ces recueils forment un corpus hybride dans les genres qu'il renferme et se situe chronologiquement avant la traduction progressive des Psaumes par Clément Marot. Ils ont acquis une place centrale dans l'élaboration et la défense d'une identité réformée, et proposent des chansons spirituelles « en marge de cette institutionnalisation de la dévotion réformée », «mise en route par l'officialisation progressive des Psaumes comme pilier de la liturgie réformée, comme livre de prières $»^{49}$. C'est en effet en septembre 1537 que Calvin suggère au Conseil que des psaumes soient chantés pendant le culte. Malgré le caractère composite de leur contenu, ces trois recueils sont vecteur d'une identité qu'il faut relier à leur chronologie propre, celle qui mène progressivement dans l'espace suisse-romand à une Église réformée implantée et institutionnalisée. Elle se constitue en résonance au rejet de la messe et s'exprime notamment dans une gestuelle liée au « renversement de l'autel $»^{50}$.

Dans l'analyse qui suit, nous souhaitons nous concentrer sur le geste d'écriture. Il s'agit d'interroger la réappropriation de genres liés à la chanson spirituelle, de questionner l'investissement de ce lieu, témoin d'une pratique d'écriture d'édification du christianisme médiéval et de saisir l'enjeu qui se dessine autour de ces formes d'expression. La chanson pieuse en langue vulgaire n'est pas

47. S'ensuyvent plusieurs belles et bonnes chansons que les chrestiens peuvent chanter en grande affection de coeur; pour et affin de soulager leurs esperitz et de leur donner repos en Dieu [Neuchâtel, P. de Vingle, 1533] : désigné par la suite : Belles et bonnes chansons, 1533.

48. Noelz nouveaulx. Musiciens amateurs des Cantiques, Au nom de Dieu, chantez noelz nouveaulx, Lesquelz sont faictz sur les vieulx et antiques : Je vous supply, delaissez les lubriques: Ne chantez point brayant comme noz veaulx, Glorieux chantres, ne vault point deux naveaux Recordez vous que Dieu veult l'humble cœur En foy contrict. Note cela chanteur, [Neuchâtel, P. de Vingle, 1534].

49. Véronique Ferrer, «La chanson spirituelle au temps de la Réforme (1533-1591) », Studia Litteraria Universitatis lagellonicae Cracoviensis, 7, z. 1, 2012, p. 43-52, cité ici p. 43 ; Christian Grosse, «L'esthétique du chant dans la piété calviniste aux premiers temps de la Réforme (1536-1545) », Revue de l'histoire des religions, t. 227, fasc. 1 : Beauté du rite. Liturgie et esthétique dans le christianisme (XVI ${ }^{e}-X^{e} I^{e}$ siècle), 2010, p. 13-31.

50. Ch. Grosse, Les rituels de la Cène, p. 69-72. 
apparue de manière spontanée avec la Réforme. Elle rejoint une pratique remontant au Moyen Âge, qui se lit notamment dans le recours au contrafactum, une technique que les trois recueils imprimés par Pierre de Vingle utilisent. Un tel procédé a d'ailleurs été employé un siècle plus tôt par Olivier Maillard pour dénoncer en prêche l'obscénité de certains chants populaires. Il découle d'un geste de réécriture et porte une intention d'édification. Il manifeste une volonté de réorienter l'attention vers Dieu, en purifiant un contenu de ses attaches aux louanges d'un amour mondain. Véhicule de l'expression d'une spiritualité et lieu d'une écriture de transmission de savoirs, la chanson entrerait à part entière dans le processus de purgation et de nettoyage menant à une foi rénovée dans sa pureté. Les modalités d'un chant communautaire sont ainsi formulées ; bien plus, la chanson comme genre est réinvestie dans son usage et sa finalité.

\section{Les « Chansons nouvelles » : DU Rejet À Une Communauté}

Le recueil des Chansons Nouvelles porte en lui et de manière quasi organique le programme identitaire d'une communauté en train de se définir. Cette nouvelle communauté, de fidèles et de prédicants, est appelée à se distancier des rituels romains envisagés comme contraires aux Écritures et édictés par la seule autorité des hommes. Elle s'ancre dans l'impératif de marquer une séparation, ou mieux, de se démarquer en refusant et en dénonçant des pratiques dévotionnelles communautaires, par la rupture de jeûnes (notamment lors du carême), l'abandon de la messe et le refus de la confession ${ }^{51}$. Le recueil invite à une mise en acte de cette séparation, une invitation qui se lit dès la page de titre et qui se développe tout au long de l'organisation interne des cinq chansons. Le compilateur encadre son ouvrage d'une référence à Matthieu 15,13, celle d'un Christ face à des Pharisiens blessés par ses propos. Aux disciples qui le lui font remarquer, Jésus répond : « Toute plantation que mon père n'a pas plantée sera arrachée ». Ainsi, l'emploi de l'attaque, s'il participe aux modes d'expression

51. William G. Naphy, « Catholic Perceptions of Early French Protestantism : the Heresy Trial of Baudichon de la Maisonneuve in Lyon, 1534 », French History, 9/4, 1995, p. 451-477. 
de cette communauté, contribue bien davantage à la formation d'une identité. L'offense est un passage obligé pour identifier les porteurs de fausses doctrines et de mauvais enseignements. Est offensé celui qui est aveugle ou encore aveuglé, autrement dit non réceptif au Christ, et donc semblable aux Pharisiens, défenseurs rigoureux de la Loi et du rituel. Toutefois, celui qui ose se détacher de ces «conducteurs d'aveugle» s'illustre par sa clairvoyance, sorti qu'il est de l'état d'aveuglement et d'ignorance. Il a en d'autres termes suivi et mis à exécution les paroles du Christ, exhortant ses apôtres à s'éloigner des Pharisiens et à les laisser dans leur erreur.

Le recueil veut exposer et démontrer la teneur des erreurs des «ministres de Satan » qui remplissent le monde. C'est ainsi très logiquement que ces Chansons Nouvelles commencent par exposer «l'origine, autorité et puissance de l'Évangile », lieu de la parole révélée et reçue par les premiers témoins du Christ ${ }^{52}$. L'Évangile se plaint de sa situation, car «l'humain entendement» rempli d'arrogance et d'audace le tourmente et a, par ce biais, occulté son sens, et méprisé, en d'autres mots, son accès. En effet «tant de faulx seducteurs, aveugles conducteurs» «cuydantz estre sage » «m'ont», dit l'Évangile, «fermé les passages ». Mais pas seulement, ils «me blasment en tous lieux, nouveau seditieux me appellent en toute place». Finalement, dans leur bouche, l'Évangile est qualifié non seulement de nouvelle foi mais surtout de doctrine erronée, montrant par ce jugement leur dédain face $\mathrm{au}$ 《 grand roy » et à «sa grâce » ${ }^{53}$. En effet, cette parole est bien le lieu du «vrai salut et vie», puisque suivre l'Évangile c'est reconnaître et servir la gloire du Christ, qui par sa mort efficace a sauvé l'humanité pécheresse ${ }^{54}$.

Ce texte se poursuit par la chanson d'une messe qui clame sa fin venue et demande à être sauvée par ses protecteurs, les implorant, pour les services qu'elle leur a rendus en richesses et autres biens mondains, de trouver une solution ${ }^{55}$. Néanmoins, la messe a l'intuition de son absence de fondement scripturaire et reconnaît dès lors qu'un seul moyen lui reste pour perdurer :

52. Chansons nouvelles, $1534, \mathrm{f}$. AAiv ${ }^{\circ}$-Aiiir ${ }^{\circ}$.

53. Ibid., f. AAiir ${ }^{\circ}$.

54. Ibid.

55. Chansons nouvelles, 1534 , $\mathrm{f}$. AAiiir ${ }^{\circ}$-AAiiiir ${ }^{\circ}$. 
la violence, préférant subir la pendaison plutôt que de devoir partager son espace «en village ou ville » avec l'Évangile ${ }^{56}$. Après la messe, le recueil passe aux prêtres qui ont usurpé ce nom de berger et de guide du peuple ${ }^{57}$. Loin d'enseigner aux fidèles, les prêtres cultivent contre eux leur autorité prise sur les Écritures et les entretiennent dans l'erreur. Les principales sont révélées : la transformation des espèces en corps et sang du Christ et la présence réelle. C'est pourquoi on exhorte ces magiciens faisant « tant de tours », fabriquant « tant des dieux que des pains », à lire « au premier Saint Luc, aux Actes et Sainct Marc au dernier», car «Jesus est à la dextre du pere tout puissant $»^{58}$. Les prêtres se font «dieux en terre » par la confession qu'ils défendent, mais ce sacrement ne vaut rien : seul le Christ sauve. Dès lors, les prêtres se doivent d'être conscients, dans une strophe écrite en résonance avec l'évangéliste Matthieu, que désormais «plus n'est vostre saison », «puisqu'estes tous plantez contre dieu et raison, Vous serez desplantéz $»^{59}$.

Parti de l'Évangile, source de vérité et de salut, dont la puissance de «glaive de Dieu» ruinera la messe, fille de Satan, et le «pape cest antechrist meschant et toute sa vermine », le recueil exploite l'argumentaire des fauteurs identifiés, à rejeter, pour opérer en fin de compte un ralliement ${ }^{60}$. En effet, il en appelle au «bon vouloir» de ces «pauvres papistes ignorants » et travaille à les convertir, les enjoignant de revenir au Christ ${ }^{61}$. Il s'agit d'amener l'auditeur ou le lecteur à une prise de conscience, son attitude «débonnaire » lui a fait cultiver l'erreur et nourrir son ignorance. Désormais, il doit s'en extraire, «il est temps» «puis que Jesus vous est presché $»^{62}$. S'ensuit une liste de gestes et d'actes dévotionnels à quitter, puisqu'ils mènent à l'idolâtrie, à une complicité avec le mensonge, et qu'ils soutiennent l'oisiveté et la «paillardise» du clergé. Le recueil suit plus encore la perspective constructive et se termine par une chanson rédigée à l'intention des «chrestiens $»^{63}$.

56. Ibid., f. AAiiiiir ${ }^{\circ}$.

57. Ibid., f. AAiiiir ${ }^{\circ}-\mathrm{AAVv}^{\circ}$.

58. Ibid., f. AAiiiiiv ${ }^{\circ}$.

59. Ibid., f. AAiiiiv ${ }^{\circ}-\mathrm{AAVr}^{\circ}$.

60. Ibid., f. AA $\mathrm{Vv}^{\circ}$.

61. Ibid., f. AA Vv-AAviir ${ }^{\circ}$.

62. Ibid., f. AAvir ${ }^{\circ}$.

63. Ibid., f. AAviir ${ }^{\circ}$-AAviiiv ${ }^{\circ}$. 
La chanson finale délivre, références bibliques à l'appui, un manuel pour une pratique chrétienne, « selon Dieu », du chant. Il faut quitter «chansons d'yvrognerie », «chansons charnelles» pour proposer au Seigneur, dans cet éloignement vécu, intériorisé et non pas seulement dit et prétendu, du monde, de ses voluptés et vanités, des «chansons spirituelles, de saincte escripture extraictes $»^{64}$. Puisque $«$ sans foy $» ~ «$ impossible est [...] de plaire à nostre sauveur », il s'agit non seulement de chanter « de bouche, mais de cœur bien plus ardemment quand me touche l'Esprit de Dieu $»^{65}$.

Le chant devient dès lors le lieu d'expression d'un cœur croyant, d'un cœur chrétien, car «si chrestiens sommes en noz cueurs porter debvons l'amour profonde de ce bon Christ $»^{66}$. Cette voix vibrante de foi en ce Christ sauveur des hommes, qui à "luy seul descharge les pecheurs $»^{67}$ est portée et soutenue par un texte intelligible, suivant le principe présent dans l'épître aux Corinthiens ${ }^{68}$. Lecteurs et auditeurs sont d'ailleurs exhortés plus haut par le poète et le chanteur après lui, d'aller lire "au quatorizième chapitre La premiere aux Corinthiens ». C'est bien là où saint Paul a indiqué comment « doibvent les vrays chrestiens prescher, prier, chanter aussi ». Comprise de tous par le recours au vernaculaire, cette « voix intelligible» permet que «fruict à tous soit perceptible». Ainsi, ce cœur en rendant gloire à Dieu «nostre bon père de cieulx [...] qui de la mort nous a tiré par Jesus qui a faict la guerre contre Satan » devient un exemple et on lui confère un but pédagogique ${ }^{69}$. En incarnant son texte, le chanteur, porteur d'une foi vive, se fait instructeur d'une pratique, celle d'un juste service à Dieu et il conduira son prochain par un geste de charité en propageant un contenu de foi et d'autorité qui vise à «louer Dieu en son office $»^{70}$. De plus, il amènera son frère et plus largement la communauté à rendre "memoire [...] de la grace » du don du Christ pour l'humanité, de sa vie de

64. Ibid., f. AAviiv ${ }^{\circ}$.

65. Ibid.

66. Ibid., f. AAviiir ${ }^{\circ}$.

67. Ibid., f. AAvir ${ }^{\circ}$.

68. Ibid., f. AAviv ${ }^{\circ}$; Aviir ${ }^{\circ}$.

69. Ibid., f. AAviiir ${ }^{\circ} \mathrm{v}^{\circ}$.

70. Ibid., f. AAviiir ${ }^{\circ}$. 
pauvreté, de «ses langueurs » et de son sacrifice ${ }^{71}$. Finalement, par ses louanges, il remerciera le Fils et le Père de leurs grâces. Cet acte de louange a ou recèle une finalité communautaire. Le chant, pratiqué collectivement, prémunit la communauté du péché d'ingratitude.

Désormais, les «pauvres papistes ignorants », en rejetant leurs anciennes habitudes dévotionnelles nées d'ordonnances humaines, deviendront de vrais chrétiens et formeront une communauté ayant, comme le suggère la dernière chanson des Chansons Nouvelles, "une fois l'année ou deux memoire seulement de la grace qu'il [Jesus] a donné à nous », se démarquant ainsi des Pharisiens aveugles et des «papistes obstinéz et blasphemateurs », sacrifiant le Christ à répétition et vendant les sacrements ${ }^{72}$. Les pièces du recueil des Chansons nouvelles attestent la progression vers la création d'une communauté. Relais de la dénonciation, elles nous confrontent à un chant mesuré dans des mélodies profanes, devenant le transport d'une émotion libératrice sous l'action de laquelle le chanteur se défait de son asservissement à des pratiques dévotionnelles et des médiateurs humains, et se joint à nouveau, par cette sortie de servitude revendiquée et exposée, au Christ, à Dieu et à ses grâces. Par ce biais, est rétablie cette relation entre le fidèle et son Dieu qu'exploitent et investissent les Belles et bonnes chansons.

\section{LES « Belles et Bonnes Chansons » : LE COMBAT DES FIDÈLES CHRÉTIENS}

Le recueil de dix-neuf chansons intitulé Belles et bonnes Chansons s'ouvre sur des conseils invitant les lecteurs chrétiens à chanter $«$ heur en bien entendant la teneur $»^{73}$. Cette teneur découle d'une attention aux « saincts escriptz », et le lecteur est appelé sous forme impérative à ne pas en «perdre la saveur». Ces conseils portent la signature de l'auteur du recueil, Matthieu Malingre, prédicateur engagé dans l'effort bernois d'évangélisation en territoire romand, qui se démasque dans ce poème d'introduction

71. Ibid., f. AAviiir ${ }^{\circ}$.

72. Ibid., f. AAviiir ${ }^{\circ}$.

73. Belles et bonnes chansons, 1533 , f. $\mathrm{AIv}^{\circ}$. 
par le double acrostiche de son nom ${ }^{74}$. Posant le cœur au centre d'autant que Jésus «n'oyt point vos voix et cryz», il exhorte ses lecteurs à le lire et à suivre cette méthode, qu'il fait sienne, certifiant «que icy enchanterie n'y ha». Cette invitation se clôt sur sa devise, construite sur un jeu d'assonances avec son nom, Malingre : Y me vint mal à gré ${ }^{75}$. Désormais poète et instructeur d'une pratique nouvelle du chant, le prédicateur laisse ici ouverte la possibilité d'un désagrément et l'occasion d'une désapprobation. Il s'en fait en quelque sorte porteur, venant se loger et se dire dans cette quasi-simultanéité de la voie juste à suivre (qu'il défend et expose à ses frères), et de l'instant de la chute qu'il incarne et réprouve tout à la fois pour lui et son prochain. Cette préoccupation se lit, à la dixième chanson du recueil, dans l'écho littéral à cette devise alors mise en contexte poétique ${ }^{76}$. Le chanteur déclare avoir mis son espérance en Dieu seul. Désormais seul secours et seul recours, il sait qu'il n'implorera que Dieu. Ainsi dit-il, «quant mon ame est en transe à toy je cours et par foy me soutiens », délaissant toutes médiations humaines, figurées par la référence aux ordres mendiants ${ }^{77}$. Il en appelle à un service et à une louange à Dieu «sans discerner de places ou de lieux $»^{78}$. Néanmoins, il se repent et se confie à ce «prince regnant $[\ldots]$ d'avoir mal faict» car, «y me vint mal à gré » ${ }^{79}$. Conscient de s'être détourné de Dieu par sa confiance donnée jadis aux hommes, il requiert le pardon de ses manquements et souhaite parvenir « en ung meilleur degré ».

74. Fils d'un gentilhomme de Normandie (Jehan Malingre, seigneur de Morvillers-Saint-Saturnin), dominicain engagé dans les ordres, il prêche dans les années 1520 à Blois contre la messe avant d'arriver dans l'actuelle Suisse romande. En 1533, il participe aux presses de Pierre de Vingle et devient pasteur d'Yverdon en 1536: L'espitre de M. Malingre envoyée à Clément Marot: en laquelle est demandée la cause de son département de France avec La responce dudit Marot, imprimé par Jean Enscheidé et fils à Harlem pour la libraire Tross, Paris, 1868; Guillaume Farel, 1489-1565 : biographie nouvelle écrite d'après les documents originaux, par un groupe d'historiens, professeurs et pasteurs de Suisse, de France et d'Italie, Neuchâtel, Delachaux \& Niestlé, 1930, p. 399-401 ; Henri Vuilleumier, Histoire de l'Église réformée du Pays de Vaud sous le régime bernois, t. 4, Lausanne, Éditions La Concorde, 1933, p. 629.

75. Venir à gré signifie, dans sa forme positive : être agréable, convenir. Ce même schéma d'encadrement poétique se trouve au tout début des $\mathrm{Noelz}$ nouveaulx, construit également sur une similarité de contenu.

76. Belles et bonnes chansons, 1533 , f. BVIr ${ }^{\circ}-\mathrm{BVIv}^{\circ}$.

77. Ibid., f. BVIr'.

78. Ibid., f. BVIv ${ }^{\circ}$.

79. Ibid. 
C'est bien autour du combat mené par le fidèle chrétien que se développe l'ensemble du recueil, en partant de la nature de l'homme, fils d'Adam et donc être de perdition, sans mérite, vil et inutile. Cet homme est aux prises avec Satan et ses tentations. Face à ces assauts, il cherche non seulement réconfort et soulagement mais aussi pardon. La miséricorde divine et sa quête constituent par conséquent la thématique du recueil, qui diffuse par ses chansons l'impératif d'une louange à la gloire de Dieu, délivrant de l'état de péché par le don suffisant et unique de son Fils, seul médiateur et intercesseur pour le pécheur et son salut auprès du Père. Le recueil privilégie une relation de cœur à cœur entre Dieu et le fidèle, renforçant sa foi tournée vers le Christ, une foi reconnaissante de l'efficacité de son sacrifice. Il accompagne le lecteur et chanteur dans sa condition pécheresse et fragile, l'amenant à se reconnaître dans cet état et l'ouvrant au secours que l'homme obtient de Dieu seul. Sur ce chemin, le combat du Christ qui «a eu victoire en la croix contre Satan» est source d'inspiration et constitue un exemple face à l'homme pécheur assailli par les œuvres du diable. Dans cette lutte de tous les instants contre la luxure, la gourmandise, la colère, l'avarice, l'homme, mis à l'épreuve dans sa foi, prouve son appartenance à Dieu. Mais l'homme, "mystique chrétien» qu'il cherche à être, doit bel et bien se faire «imitateur de Jesus Christ» et, ce faisant, doit plus encore que «ces pharisiens évite[r] les erreurs » et se défaire de l'Antéchrist couronné d'or, portant «orgueilleux habits » et vivant agréablement, car ainsi il renonce à être son serviteur et se met au service du Christ et de son Évangile ${ }^{80}$. On note, dans ce recueil, une distorsion du temps, lisible déjà dans les accents polémiques du recueil précédent, où les fidèles chrétiens se trouvent confrontés aux acteurs présents pendant la vie du Christ. La chronologie se confond et les Pharisiens, hypocrites, deviennent les « caphards », frères mendiants, docteurs en théologie et prêtres vendeurs de sacrements ${ }^{81}$.

Le soulagement promis dès la page du titre aux chanteurs et aux lecteurs se loge dans le cœur, un cœur venu à Jésus, car « de

80. Dans les Belles et bonnes chansons se rapporter sur cette thématique aux chansons : $11\left(\right.$ f. $\left.\mathrm{BVIv}^{\circ}-\mathrm{BVIIIv}^{\circ}\right) ; 8$ (f. $\mathrm{Biiir}^{\circ}-\mathrm{Biiiv}^{\circ}$ ), plus particulièrement f. Biiir $^{\circ}$; 9 (f. BIIIV $\left.{ }^{\circ}-\mathrm{BVIr}^{\circ}\right)$.

81. Ibid., f. $\mathrm{BIv}^{\circ}-\mathrm{BIIr}^{\circ}$. 
luy tout seul, nostre salut distile $»^{82}$. Dès lors, par cette adhésion présentée dans la troisième chanson du recueil, le chanteur ou le lecteur sera fait membre de la famille du Christ et attaché à Dieu. Quant à son péché, il «luy est tout condonné et pardonné, par foy tout est loisible ${ }^{83}$. C'est pourquoi, le poète et le chanteur avec lui exhortent leur auditoire à y réfléchir, l'apostrophant en ces termes: "pense a cela et croy homme labile $»^{84}$. Mais cette démarche de foi est loin de se faire dans la passivité de la récitation et de la réception de cette vérité rendue par ces strophes qui ont été rédigées, comme l'ensemble du recueil à suivre la page de titre, au nom de Dieu et composées « au plus pres de l'esprit de Jesus Christ, contenu ès sainctes escriptures ». Elle nécessite une implication, une prise en main par laquelle auditeur, chanteur ou lecteur examine, à la demande du poète, «ce que, dit-il, j'ay ramené en la chanson, s'il est bien retourné [rendu] et entendu, il t'est sainct et utile $»^{85}$. Le chanteur est investi d'un rôle en même temps qu'il examine un contenu de foi, il proclame et dit sa foi. Il devient par la déclamation d'un texte garanti dans la justesse de son contenu et formulé par un poète-prédicant, transmetteur et «enseigneur» de vérité. Dans sa valeur performative, le chant détient ici une valeur d'agent créateur d'une communauté de vrais chrétiens, dessinant au sein même d'un corps social jusque-là unifié, une assemblée nouvelle ou un chœur d'hommes et de femmes porteurs d'une foi vive nourrie à l'espérance de salut en Christ uniquement. Désormais, ce chœur transmet et défend des pratiques rituelles et plus largement dévotionnelles épurées et rénovées. Dès lors, le chant sert à l'établissement d'une culture religieuse nouvelle qui s'affirme dans un rapport redessiné de l'homme à sa nature pécheresse, à son chemin de salut, à ses louanges au Père et enfin à ses liens aux médiateurs de sa salvation. Cette rénovation se transcrit dans le recours au Christ seul, sans l'intermédiaire des hommes. Cette culture nouvelle se revendique aussi dans la conception d'une cène comprise dans sa dimension

82. Ibid., f. AVIv'.

83. Ibid., f. AVIIr ${ }^{\circ}$.

84. Ibid., f. $\mathrm{AVIv}^{\circ}$.

85. Ibid., f. AVIIr ${ }^{\circ}$ : «Viens a Jesus, foy t'en apprend le stile. Chante d'esprit quant tu coux ou tu file. Et note bien, ce que j'ay ramené En la chanson, s'il est bien retourné Et entendu, il t'est sainct et utile. » 
commémorative et d'un Noël rénové dans son sens et dans sa célébration $^{86}$.

\section{LES « Nö̈LZ NOUVEAULX » : UNE DÉVOTION AJUSTÉE}

Avec les Noelz nouveaulx imprimés par Pierre de Vingle, la critique de Noël se lit à l'échelle d'un genre précis auquel le titre vient délibérément se référer ${ }^{87}$. Le recueil s'apparente, par son titre et sa thématique, à une tradition liée aux festivités de la Nativité. Dans la chrétienté médiévale, la célébration de Noël connaît l'émergence de pièces jouées, de dialogue entre le célébrant et l'assemblée, de chants liturgiques, d'hymnes et de tropes. Elle est notamment liée à la production de recueils de Noëls populaires, appelés Noëls ou Noëls nouveaux, où viennent s'entremêler chansons spirituelles et chansons de fêtes célébrant les joies d'un banquet ${ }^{88}$. Ainsi, dans l'investissement de ce genre autant qu'au niveau du contenu par le rejet de la dévotion mariale et les critiques du calendrier liturgique, se loge un acte de subversion à des fins religieuses. Il se comprend dans la nécessité de réorienter et de rénover une pratique - la célébration de Noël - de la vider

86. Se rapporter aux Chansons nouvelles, $\mathrm{f}$. AAviiiir ${ }^{\circ}$, où le poète-chanteur suggère encore que la cène, conçue comme un temps de mémoire du dernier repas de Jésus, ne soit célébrée que deux ou trois fois l'an.

87. Les recueils de Noëls imprimés en France au XVI ${ }^{\mathrm{e}}$ siècle ont été recensés par Adrienne Fried Block, The early French parody Noël, Ann Arbor (Mi.), UMI Research Press, 1983, qui dresse également les caractéristiques du genre, son origine et les usages religieux et musicaux. Le recueil publié par Pierre de Vingle, imprimeur d'origine lyonnaise, doit beaucoup à l'existence de la tradition de ces Noëls chantés qui sont largement imprimés à Lyon dans les années 1520. L'imprimeur lyonnais et beau-père de Vingle, Claude Nourry en publie d'ailleurs deux. À ce propos, on peut consulter les analyses complémentaires et détaillées suivantes qui relient les Noëlz Nouveaulx de 1533 aux noëls catholiques mais plus largement aux usages et pratiques du chant au début du Xvi ${ }^{\mathrm{e}}$ siècle : Amélie Aubut, «Les Noelz notés de Moderne et les Noelz nouveaulx de Vingle. Étude sur un transfert de traditions»; et Jean Duchamp, «Les Noelz nouveaulx de Malingre (Vingle, vers 1533) : étude d'un transfert de traditions », Les imprimés réformés de Pierre de Vingle (Neuchâtel, 1533-1535), dir. D. Desrosiers-Bonin et W. Kemp, Littératures, n 24,2007 , p. $95-126$.

88. Sur les traditions et festivités liées à la Nativité : Arnold Van Gennep, Manuel de folklore français contemporain : de Noël aux rois. 1. 8. Cycle des douze jours, Paris, A. et J. Picard, 1988, p. 3205-3268 ; Amédée Gastoué, Noël, origines et développements de la fête, Paris, Bloud, 1908, p. 44-56. 
complètement de son sens et de la réinvestir d'une signification neuve découlant d'une lecture des ressources scripturaires.

Proposant une relecture de ce temps menant à l'Incarnation de l'Enfant Jésus appelé à se sacrifier pour l'humanité, les Noelz nouveaulx soulignent la suffisance et l'unicité de cette naissance. Ils contiennent en sous-main la critique d'un temps structuré et occupé tout au long de l'année à réitérer le chemin menant au sacrifice du Christ. Ils viennent ainsi toucher une thématique profondément liée à la lecture par l'Église traditionnelle de la célébration eucharistique comprise comme un instant de transsubstantiation où le Christ descend du ciel et prend à travers les mains du prêtre littéralement corps dans les espèces consacrées $^{89}$. C'est pourquoi, le recueil émerge certainement d'un contexte d'intervention précis mis en place par Guillaume Farel dès septembre 1532 à Genève. Là, entre 1533 et 1534, les prédicants prennent pour cible la messe de Noël et tentent d'empêcher sa célébration ${ }^{90}$. Dans cette perspective, loin d'être un choix arbitraire, l'ouvrage De la tressaincte Cene qu'on chante communément - traité contemporain des Noëlz nouveaulx attribué à Farel et au jeune Viret - choisit de procéder à un exercice de décryptage exposant et déroulant la signification de l'ensemble gestuel et verbal de l'ordinaire de la messe en partant de la messe de Noël, qui a valeur d'exemple ${ }^{91}$.

Réorientation du regard et dynamique de renouvellement trouvent une expression constante dans l'emploi du contrafactum, venant s'inscrire d'entrée de jeu dans la première pièce du recueil et toucher l'hymnologie spécifique à Noël : l'hymne de l'Avent,

89. Les mains du prêtre sont envisagées comme l'utérus de la Vierge dans le manuel défendant la dignité des prêtres et curés : Stella clericorum, éd. par Eric H. Reiter, Stavelot, Éditions Wavremont, Toronto, Pontifical Institute of Medieval Studies, 1997, p. 40-41.

90. Sur l'action des prédicants, se rapporter à Ch. Grosse, Les rituels de la Cène, p. 54 ; Jeanne de Jussie, Petite Chronique, Einleitung, Edition, Kommentar von Helmut Feld, Mainz, Verlag Philipp von Zabern, 1996, p. 116-117, 153-154.

91. L'écrit daté de 1532 casse la dimension sacrificielle présente dans la célébration eucharistique et rejette du même coup la présence réelle du Christ dans les espèces consacrées. Cette lecture est jugée fautive et garante de la puissance cléricale: [Guillaume Farel], De la Tressaincte Cene de nostre Seigneur Jesus : et de la Messe qu'on chante communement, publié et introduit par Francis M. Higman : «Les débuts de la polémique contre la messe », p. 264 (f. D8r ${ }^{\circ}$ ). 
Conditor alme siderum. Dans cette traduction libre en vernaculaire transposé plus tôt en 1523 en allemand par Thomas Müntzer dans une louange à Dieu, créateur de toutes les étoiles, le poète se centre ici essentiellement sur Jésus, « Aigneau de Dieu tresprecieux » né de la Vierge et «juge avenir » étant « seul qui peux subvenir à tes servantz $»^{92}$. Le Christ «veut sauver en grande douceur tous les pécheurs repentis en cœur » et ce par la grâce seule ${ }^{93}$. C'est certes ce message univoque que délivre le recueil des Noelz nouveaulx, mais par lui, les lecteurs sont davantage amenés à entrer dans la réelle importance de Noël, celle justement de cette purgation de l'âme rendue possible par l'incarnation et la passion du Christ. La naissance du Sauveur correspond à l'entrée dans la Nouvelle Alliance et annonce le règne de l'Évangile. Le recueil se divise alors en trois thématiques se calquant sur trois temps : l'attente de l'avènement du Messie et son annonce, l'incarnation du verbe divin et enfin le partage de l'avènement et les signes de sa présence sur terre. Il met tout particulièrement en évidence trois figures centrales : Marie, Jésus et les témoins, bergers et mages. Une place particulière est tout d'abord allouée à Marie, siège de l'incarnation puisque son ventre est le réceptacle du fils de Dieu. Par son obéissance au plan divin, elle offre à Jésus une filiation dans la lignée davidienne et permet de suivre les prophéties messianiques. Il faut donc exalter son rôle de servante respectueuse et soumise à la volonté divine. Elle est dès lors «au ciel» cette «dame haultaine », cette «dame qui ayme Dieu de meilleur cœur» que personne n'égale, et « qui en dit mal ou la diffame » « est meschant et menteur $»^{94}$. Sa foi en Christ lui a ouvert le repos en Dieu. Marie devient la figure des chrétiens, semblable à eux, leur salut ne devant être attendu que par le Christ seul. En effet, poursuit le poète « il n'y a ny sainct ny ange qui fut jamais Emmanuel », étant bien le seul à être mort pour l'homme et à œuvrer pour son salut,

92. Noelz nouveaulx, 1534, f. AIIr ${ }^{\circ} \mathrm{v}^{\circ}$; «Gott, heiliger Schöpfer aller Sterne », in : Evangelisches Gesangbuch. Ausgabe für die Evangelische Kirche in Hessen-Nassau, Leipzig, 2004, Nr. 3. La présence du Conditor alme siderum comme pièce d'ouverture au recueil de Pierre de Vingle dénote une volonté de s'inscrire dans la tradition des anthologies de noëls de l'époque: J. Duchamp, «Les Noelz nouveaulx de Malingre (Vingle, vers 1533) : étude d'un transfert de traditions », p. 109.

93. Noelz nouveaulx, $1534, \mathrm{f} . \mathrm{AVv}^{\circ}$.

94. Ibid., (chansons 4 et 5), f. $\mathrm{AVr}^{\circ}, \mathrm{AVIr}^{\circ}$. 
à la différence de Marie, cette servante d'excellence et de tous les saints ${ }^{95}$. Il s'agit donc d'observer et de mettre en pratique l'attache de la mère au Fils de Dieu, engendré en tant qu'homme mais avec une divinité cachée sous cette apparence. Le Christ a pris nature humaine dans la précarité d'une crèche, s'est enfermé dans le temps mais a vaincu la mort et Satan pour sauver l'humanité de sa déchéance adamique, avant d'être assis en souveraineté auprès du Père.

L'homme chrétien se doit de célébrer cet événement en admirant cette nativité, lieu d'expression de "la grande humilité du filz de Dieu, vray roy ». De ce geste d'amour découle la soumission du chrétien à ce seigneur, qui doit occuper seul «quand estes a repos », tel que le conseille le poète à « Robin et toy Denyse » « voz communs propos ${ }^{96}$. Cette soumission conduit les fidèles à observer Bethléem - lieu de naissance du Sauveur dans sa pauvreté - et à se défaire des joies mondaines, un renoncement auquel mènent également les circonstances de la montée du Christ au ciel sans gloire ou «pourpre $»^{97}$. Le fidèle et plus encore les «bergiers, seigneurs, prelatz» doivent tirer un enseignement de la contemplation de la vie et naissance du Christ, ainsi «en contemplant le Christ, Mettez son faict et le vostre en balance Et vous verrez qu'aucuns sont l'antechrist $\gg^{98}$. Dans cette dévotion tournée vers le Christ, lumière du monde et prince de la paix, il ne faut pas manquer de rendre grâce à Dieu, ce Père aimant, qui a donné son propre fils, assurant le rachat de l'humanité entière une fois pour toutes.

Les Noelz nouveaulx ciblent le service rendu à Dieu, dans les prières et les louanges. Le recueil s'ouvre d'ailleurs sur cette injonction par la référence au psaume 113, transposée dans ce devoir des «humbles enfants», serviteurs de Dieu, de mettre du soin à louer le très Haut pour avoir honneur et Malingre, présent

95. Ibid., f. $\mathrm{AVIv}^{\circ}$; se reporter également à la huitième chanson du recueil : « car Jesus est né, Motz veridiques sans luy tout est damné. Grâce à luy rendons, de cœur, de voix et d'ame, Et salut attendons de luy : car c'est blasphème de Dire que femme puisse donner repos. $\mathrm{C}^{\prime}$ il est infame qui soustient ces propos » (f. $\mathrm{Bv}^{\circ}{ }_{-}$ $\left.\mathrm{BIIr}^{\circ}\right)$.

96. Ibid., (chanson 6), f. AVIIv ${ }^{\circ}$.

97. Ibid., (chanson 15), f. BVIIIv ${ }^{\circ}$.

98. Ibid., (chanson 20), f. $\mathrm{CVv}^{\circ}-\mathrm{CVIr}^{\circ}$. L'idée est reprise dans la chanson 22 : f. CVIv ${ }^{\circ}$-CVIIr ${ }^{\circ}$. 
en acrostiche, de préciser la méthode, poussant chanteurs et musiciens à chanter «noelz nouveaulx, lesquelz sont faictz sur les vieulx et antiques », les suppliant finalement de s'éloigner des «lubriques $»^{99}$. Ces exigences trouvent des résonances à l'intérieur du corpus, où le poète et le chanteur à sa suite affirment leur intention de chanter Noël « en la foy bien appris », et convainquent après un récit de la vie du Christ «Robin, Jaquet et Magdeleine » de désormais privilégier "sainctz accordz et sans voix dissolue » de ne plus chanter « chanson ordre et pollue », synonyme de «pleur perpetuel $»^{100}$. S'il s'agit de louer « un seul Dieu, auquel on doibt gloire et honneur», il convient surtout de chanter Noël «par la Parolle de Jesus, car toute creature ment, fors que luy seul qui est la sus $»^{101}$. Cette crainte de l'homme mêlée à l'obligation de fêter la Nativité en conformité avec la vérité scripturaire esquisse le devoir du pasteur, une figure définie par le rôle de témoins des mages et des bergers, venus à l'Enfant nouveau-né et devenus annonciateurs de l'avènement de la Nouvelle Alliance par la naissance de ce roi attendu et sauveur du monde. Ainsi, l'analogie avec les bergers sert logiquement à cultiver le soin délivré au troupeau, rappelant aux pasteurs qu'il faut nourrir leurs moutons « du pain qu'on faict et fondé des sainctz escriptz [...] car tout le mal qui au brebis abonde, souvent provient du pasteur ${ }^{102}$. C'est pourquoi, il s'agit pour les pasteurs de «sortir parolle immunde» de leurs bouches, mais avant tout de se libérer de la servitude de l'idolâtrie et de quitter, comme l'exhorte le poète, « voz dieux de boys $»^{103}$. Par un «nous » communautaire, le poète-chanteur exhorte les pasteurs à changer «propos, c'est trop chanté d'amours. Chantons à Dieu autre cantique » ${ }^{104}$. Les « gentilz pasteurs » sont appelés à réciter et à faire réciter «melodieusement femmes, enfantz et serviteurs » le Notre Père, car «en sainct Matthieu expressement il est escript $»^{105}$. Dès lors, c'est «le roy David» et ses psaumes, guide dans la manière «comme on doibt Dieu magnifier » qu'il faut suivre, retournant

99. Ibid., page de titre, f. $\mathrm{AIv}^{\circ}$.

100. Ibid., (chanson 16), f. CIIr ${ }^{\circ}$.

101. Ibid., f. AIIv ${ }^{\circ}$. L'idée est reprise dans la chanson 4 : f. $\mathrm{AVr}^{\circ}-\mathrm{AVv}^{\circ}$.

102. Ibid., (chanson 12), f. $\mathrm{BVr}^{\circ}-\mathrm{v}^{\circ}$.

103. Ibid.

104. Ibid., (chanson 20), f. $\mathrm{CVr}^{\circ}$.

105. Ibid., (chanson 11), f. BIIIIr ${ }^{\circ}$. 
l'honneur «non pas à nous », pasteurs, « mais à ton nom », Dieu ${ }^{106}$. À partir de cette dix-huitième chanson, se succède la transposition poétique et musicale de cinq autres psaumes, rendant visible dans le matériel employé l'appel à un retour aux ressources scripturaires dans une visée de rénovation du service à Dieu, auquel participe les récits des évangélistes, mais aussi l'attache aux chants du Psalmiste.

Dans une écriture jonglant entre le «Je» poétique, le « Tu» d'adoration, le « Nous» collectif et identitaire et le «Vous» de la dénonciation, le recueil des Noelz nouveaulx vient faire exister une communauté fondée sur une foi commune dans la réalité de la naissance du Sauveur et la promesse salvatrice de sa Passion à venir. Marie, pièce maîtresse pour ainsi dire de l'Incarnation du Sauveur ne participe pas au destin individuel et particulier des hommes en quête de salut. Elle est l'exemple suprême d'une croyance en conformité à la Nouvelle Alliance par sa reconnaissance du Fils et sa soumission au Père. À suivre le poète-chanteur de la quatrième chanson des Noelz nouveaulx, la célébration de la Nativité du Christ devrait finalement se faire tous les jours de la semaine au vu de son importance, se défaisant d'un Noël en usage dans l'Église romaine et attaché à un calendrier précis ${ }^{107}$. Enfin, par la nature même du texte composé avec une finalité de déclamation, le recueil cherche à créer l'effet d'une communauté vivante et existante $^{108}$. Sont permises l'ouverture $\mathrm{du}$ « Je» poétique à un « je » pluriel et l'assimilation identitaire d'un «nous », poétique et fictif, à un «nous » relié à une réalité collective et effective. Pour ajouter à cette dynamique, le poète des Noelz nouveaulx s'adresse dans ses exhortations fraternelles et pastorales, à Robin, Denise, Margot, Magdeleine, et Jacquette, noms fictifs sans aucun doute, mais communs à l'époque. À Roger et à Thomin est demandé d'aller chercher leur voisine «car chacun doit être enseigneur de vérité », transférant les préoccupations pastorales à l'échelle communautaire $^{109}$. Le fidèle est ainsi impliqué dans une diffusion

106. Ibid., (chanson 18), f. CIIIv ${ }^{\circ}$.

107. Ibid., (chanson 4), f. $\mathrm{AVv}^{\circ}$.

108. Ibid., (chanson 7), f. AVIIr ${ }^{\circ}-v^{\circ}$; (chanson 12), f. BVr ${ }^{\circ}-v^{\circ}$; (chanson 15), f. BVIIv ${ }^{\circ}-\mathrm{Cr}^{\circ}$. Il s'agit de personnages fréquents de la littérature populaire et qui incarnent, dans la tradition des noëls catholiques, le rôle des bergers : A. Aubut, «Les Noelz notés de Moderne et les Noelz nouveaulx de Vingle », p. 100.

109. Noelz nouveaulx, 1534, f. $\mathrm{AVv}^{\circ}$. 
et une exposition de la foi. Il participe en somme à l'effort pastoral qui est facilité et favorisé par la forme poétique choisie.

Chansons pieuses et écriture théâtrale sont des formes de l'édification médiévale et des lieux d'exercice d'une médiation du savoir, de transmission d'une spiritualité et d'un contenu de foi. Par l'emploi délibéré d'un cadre discursif connu et attendu du public, les premiers prédicants, devenus auteurs et actifs autour des presses de Pierre de Vingle, exploitent les attentes liées à un genre. Ils investissent consciemment théatre et chanson dans une dynamique de renversement des usages grâce à laquelle ils entendent gagner leur autorité, en vue de la quête d'une charge d'enseignement de la communauté. Ils proposent aux lecteurs et auditeurs, par le relais qu'offre le contenu, une médiation rénovée aux hommes, transmetteurs de la foi. Cette médiation se construit dans une observance de l'Écriture et une filiation désormais ancrée dans le seul Christ et dans son Père. Le texte dialogué du théâtre et rimé des chansons devient l'aboutissement et le résultat d'un geste réfléchi d'écriture. Il résulte d'une pratique, celle de la mise en mots, supposant un geste de formulation et un acte rédactionnel ajoutant à l'autorité de l'Écriture celle du poète-prédicant, du compositeur qui a su se laisser inspirer par le sens de l'Écriture et le transmettre sans autorisation ou sans légitimation autre que la nécessité d'enseigner. À travers la matière textuelle, se trouve ainsi exprimée la réalité d'un sacerdoce universel maîtrisé, contrôlé et encadré par une figure pastorale certes en voie de définition au niveau institutionnel, mais s'affirmant dans un travail d'énonciation d'un contenu de foi à des fins d'édification communautaire. Ces prédicants-auteurs s'exposent, autant que le contenu de leurs productions, au seul jugement des Saintes Écritures. La pratique de ce jugement revient également et prioritairement au lecteur rendu responsable de son salut et à même de discerner, en scrutant les ressources scripturaires, le vrai du faux serviteur de la Parole de Dieu. Dans cette perspective, l'investissement de ces genres connus de l'écriture religieuse participe à la responsabilisation des fidèles devant leur rédemption. Il permet la construction de nouvelles figures d'autorité : les Écritures, le prédicant mais aussi les fidèles. Bien plus, il vise une réappropriation par les fidèles eux-mêmes des instruments de leur instruction, des outils 
et des acteurs préposés à une édification communautaire, mais aussi de leur place dans la transmission d'une foi vraie et vive. Ainsi, loin d'être uniquement un moyen de diffusion des idées et un outil polémique, le recours au théâtre et au chant recèle une visée religieuse et édificatrice en soi. Il participe à part entière à la création d'une communauté nouvelle, rénovée et purifiée dans son instruction, détachée désormais de l'univers rituel de l'Église romaine et réunie autour d'un prêcheur de la Parole de Dieu dans l'attente de la cène.

grossgenevieve@gmail.com 